\title{
High frequency ventilation and respiratory distress syndrome: do we have an answer?
}

\author{
The December 1996 issue of Pediatrics carries a quotation from an article by $\mathcal{F} R$ Platt: \\ [the crucial question] ... consists of asking in your own mind, on hearing a scientific experiment described: "What \\ hypothesis does your experiment disprove?". ${ }^{1}$
}

In an issue which also carries three papers relating to high frequency ventilation this would seem a useful standpoint from which to assess these three important contributions. The areas addressed by these papers are important - areas where technology has advanced dramatically and sales of machines seem to be high-yet good evidence of efficacy is perhaps still awaited, or is it provided by these submissions?

High frequency ventilation may be achieved using either: a rapid firing jet which is positioned at the end of an endotracheal tube; an oscillator in which rapid alternation of positive and negative pressure are applied to the lungs; or by a high frequency flow interrupter which works like a conventional ventilator but at high speed. There are no randomised data to support the use of the latter technique, although at least one trial is under way. Of the three oscillators available in the UK at present, only the Sensormedics $3100 / 3100 \mathrm{~A}$ has been used in randomised trials. High frequency jet ventilation (HFJV) has not found wide acceptance in the UK.

The three papers are related by their investigation of the effects of prospective management of respiratory distress syndrome using high frequency ventilation delivered either by jet or oscillator. Most authorities agree that from their own personal experience high frequency ventilation is a valuable addition to "rescue" treatments for children in respiratory failure that is unresponsive to conventional treatments, and that its effect may be additive with nitric oxide. Its use in rescue mode may be dramatic for some patients but it is not a modality to be used lightly. In my experience at least 12 months of frequent use in a tertiary centre was needed before I felt comfortable with the use of high frequency oscillatory ventilation (HFOV) and as much harm as good may come from its untutored use: it is not a modality every hospital should have unless it can be shown to have beneficial effects in a common condition, which would then warrant wholesale training and application of the technology.

Wiswell and colleagues from Philadelphia ${ }^{2}$ report a randomised trial of HFJV vs conventional ventilation (CV) in 73 preterm infants $<33$ weeks of gestation, $>500 \mathrm{~g}$ birthweight requiring ventilation during the first 24 hours for respiratory distress syndrome. At entry peak inspiratory pressure was $>16 \mathrm{~cm} \mathrm{H}_{2} \mathrm{O}$ and $\mathrm{FIO}_{2}>0.30$. Babies were maintained on the randomised mode for seven days or until extubation or crossover criteria were met. The two groups were well matched by the randomisation process over a range of variables including pre-trial ventilator and blood gas settings, and the population comprised a similar profile to that which most UK units would expect: mean birthweights being $961 \mathrm{~g}$ and $930 \mathrm{~g}$ and gestations 26.9 and 26.6 weeks, respectively, for the HFJV and CV groups.
HFJV, as applied, did not improve respiratory variables (air leaks, need for oxygen, or ventilation at 36 weeks, days in oxygen, days of ventilation) or hospital stay. However, $31 \%$ of the HFJV group surviving to 21 days had cystic periventricular leucomalacia (PVL) compared with $6 \%$ in the CV group ( $p=0.022)$, and overall poor outcome (death, cerebral parenchymal haemorrhage, cystic PVL) was found in $48 \%$ of the HFJV group compared with $19 \%$ of those receiving $\mathrm{CV}$. The trial was halted prematurely by the data monitoring committee after 73 of a projected 194 children had entered the trial because of the dramatically worse outcome for the HFJV group. Both PVL and a poor outcome were independently related to jet ventilation.

On the face of it, there seems to be little support for the contention that HFJV is of benefit in the treatment of moderate to severe respiratory distress syndrome. The trial appeared well structured and carefully controlled. It may have underestimated the true disadvantage of HFJV as $31 \%$ of CV children crossed over to HFJV and this group included all the CV babies with cystic PVL. The authors state that they will continue to use HFJV for rescue treatment but this study would seem to offer little encouragement to introduce this technology de novo.

The results of this HFJV trial seem to echo those of the $\mathrm{HiFi}$ study published in $1989,{ }^{3}$ in which there was an increase in poor neurological outcomes and in subsequent disability ${ }^{4}$ associated with HFOV. This first study was heavily criticised on methodological grounds, mainly in its apparent "low volume strategy," and smaller subsequent "high volume" studies seemed to show benefit. The results of the Provo trial ${ }^{5}$ are therefore of great interest.

This study, involving three units, randomised 125 babies $<35$ weeks gestation receiving ventilation for respiratory distress syndrome and an arterial:alveolar oxygen ratio of $<0.50$. Study entry followed surfactant administration and the two groups were well matched over a range of pre-treatment variables. HFOV was provided by the Sensormedics 3100 or 31 00A, a powerful diaphragm driven dedicated oscillator, and $\mathrm{CV}$ by the Seechrist IV-100B.

This study seems to show impressive benefits from HFOV: less use of inotropes, less surfactant, better oxygenation over the first seven days, less oxygen and ventilator support, less treatment failure, better survival without chronic lung disease at 30 days, less oxygen use at discharge, less necrotising enterocolitis and lower hospital costs. However, even given the severity of illness at entry, in the study children adverse outcomes seem relatively common, for example, half of the HFOV and all the CV children were ventilated for more than 28 days. At discharge, $33 \%$ of the HFOV and $49 \%$ of the CV groups were receiving oxygen but accounted for by for the elegantly described relative hypoxia due to altitude. 
In terms of neurosensory outcomes, there were fewer hearing test failures in the HFOV group and, although the rates of cerebral ultrasound abnormality or retinopathy did not differ in the two groups, numerically there were fewer in the HFOV group.

The Provo study therefore seems to offer prima facie evidence for the benefit of HFOV applied within 2 to 3 hours of birth. In contrast to the HFJV study, these children were relatively more mature (mean gestation 30.9 weeks) and heavier (birthweight $1.51 \mathrm{~kg}$ ), came from a relatively affluent white population, and included few extremely low birthweight $(21 ; 16.8 \%)$ or small for gestational age children. As acknowledged by the authors, the results must therefore be interpreted with caution.

Where there are uncertainties and multiple studies, some attempt may be made to explore the effects of intervention with meta analysis. Bhuta and Henderson-Smart have reported an analysis of elective trials of HFOV, although only in abstract. ${ }^{6}$ They suggest no effect on mortality (event rate ratio (ERR): 1.05 (99\% Cl: 0.53-2.08)), but a beneficial effect on chronic lung disease (ERR: 0.55 (99\% $\mathrm{Cl}: 0.33-0.90)$ ) in those studies which used the now conventional "high volume strategy."

Clark and colleagues ${ }^{7}$ reviewed nine prospective trials of high frequency ventilation in relation to the frequency of cerebral ultrasound findings. Four of these are trials of HFJV and five HFOV and two recruited relatively late $\left(21^{8}\right.$ and $49^{\circ}$ hours of age, respectively). The wisdom of aggregating results from two different methods of ventilation is questionable. Overall, PVL and haemorrhagic lesions were consistently over represented in the high frequency ventilation group. However, these results are biased by the inclusion of the $\mathrm{HiFi}_{\text {study }}{ }^{3}$ (excess of severe haemorrhages) and that of Wiswell and colleagues ${ }^{2}$ (excess of cystic PVL), an excess of non-clinically important haemorrhages in the HiFO study, ${ }^{8}$ and differences in the definition of PVL. In the HFOV meta analysis " ${ }^{6}$ high volume strategy" trials had no excess of haemorrhages (ERR: $1.19(99 \% \quad \mathrm{Cl}$ : $0.79-1.79))$, severe haemorrhage, $(1.01(0.43-2.42))$ or PVL (0.74 (0.21-2.61))

None of these articles convincingly disproves their primary null hypothesis in a manner that is easily assimilated into clinical practice. Studies of the role of high frequency ventilation in respiratory distress syndrome span 10 years, in which time many changes have occurred in neonatal practice. Only four studies (three using HFOV) have included babies treated with surfactant; antenatal steroids have not been in wide use until recently and few studies have included a significant number of extremely preterm infants. Furthermore, only two have randomised babies in the first few hours after birth, as a true prospective evaluation. There are compelling animal data to support the very early use of HFOV using a "high-volume" strategy. ${ }^{10}$ Instituting HFOV immediately after birth (or at least on admission to the neonatal unit) is practicable and seems not to be associated with major problems. ${ }^{11}$ Such a policy has the potential to minimise surfactant loss after birth and to maximise the benefit in terms of chronic lung disease and resource use. Given that the oscillators currently available in the UK have not been used in trials, the current proposal for a UK based study randomising children to HFOV or $\mathrm{CV}$ on admission from the delivery suite (Dr S Calvert, personal communication) would seem to be timely and to have the potential to provide important evidence on which ventilator policy for the millennium can be based.

Department of Child Health

NEIL MARLOW

University of Nottingham

Queen's Medical Centre

Nottingham NG7 2UH

1 Platt J R The Step to Man. New York, NY: John Wiley, 1966.

2 Wiswell TE, Graziani LJ, Kornhauser MS, et al. HFJV in the management of respiratory distress syndrome is associated with greater risk for adverse
ons outcomes. Pediatrics 1996; 98:1035-43.

3 The HiFi study group. HFOV compared with conventional mechanical ventilation in the treatment of respiratory failure in preterm infants. $N$ Engl $F$ Med 1989;320:88-93.

4 The HiFi Study Group. High-frequency oscillatory ventilation compared with conventional intermittent mechanical ventilation in the treatment of respiratory failure in preterm infants: neurodevelopmental status at 16 to 24 months of postterm age. $\mathcal{F}$ Pediatr 1990;117:939-46.

5 Gerstamann DR, Minton SD, Stoddard RA, et al. The Provo multicentre early high frequency oscillatory ventilation trial: improved pulmonary and clinical outcome in respiratory distress syndrome. Pediatrics 1996;98: 104457.

6 Bhuta T, Henderson-Smart DJ. Meta-analysis of elective high frequency oscillatory ventilation in preterm infants with pulmonary dysfunction. oscillatory ventilation in
Pediatr Res 1996; 39:326A.

7 Clark RH, Dykes FD, Bachman TE, Ashurst JT. Intraventricular haemorrhage and high frequency ventilation: a meta-analysis of prospective clinical trials. Pediatrics 1996; 98:1058-61

8 The HiFO Study group. Randomised study of high frequency oscillatory ventilation in infants with severe respiratory distress. I Pediatr 1993;122:609-19.

9 Keszler M, Donn SM, Bucciarelli RL, et al. Multicentre controlled trial comparing high-frequency jet ventilation and conventional mechanical ventilation in newborn infants with pulmonary interstitial empysema. $\mathcal{F}$ Pediatr 1991;119:85-93.

10 Meredith KS, deLemos RA, Coalson JJ, et al. Role of lung injury in the pathogenesis of hyaline membrane disease in premature baboons. $f$ Appl Physiol 1989; 66: 2150-8.

11 Mannix PA, Costeloe K, Roper J. High frequency ventilation is a feasible method of ventilation from admission in the very preterm infant. Proceedings of the British Paediatric Association Annual Meeting 1996; 68:54 (Abstract G65). 